\title{
Evaluating the management of anaphylaxis in emergency departments: a survey in two French regions
}

\author{
${ }^{1}$ Regional Institute for Allergic and Environmental Diseases, Metz Regional Hospital, Metz, France \\ ${ }^{2}$ University of Strasbourg, France \\ ${ }^{3}$ Chest Diseases Department, Strasbourg University Hospital; University of Strasbourg, France \\ ${ }^{4}$ Department of Anesthesiology, Strasbourg University Hospital; University of Strasbourg, France
}

\section{KEYWORDS}

anaphylaxis; management; epinephrine;

emergency departments; guidelines

\author{
Corresponding author \\ Sébastien Lefevre \\ Regional Institute for allergic and environmental \\ diseases - Clinical Immunology \\ Metz Regional Hospital \\ 1 allée du chateau, \\ 57085 Metz, France. \\ Phone: +330387553383 \\ E-mail: s.lefevre@chr-metz-thionville.fr
}

\section{Doi}

10.23822/EurAnnACI.1764-1489.83

\section{To the editor}

Anaphylaxis is defined by the EAACI as a severe, life-threatening generalised or systemic hypersensitivity, characterised by its rapid onset with life-threatening airway, breathing and/ or circulatory problems (1). Epinephrine represents the treatment of choice in anaphylaxis, with intra-muscular injection being recommended upon reaching grade 2 of the Ring and Messmer severity scale (1-3). To assess both the diagnosis and management of anaphylaxis in emergency departments (EDs), we performed a study from November 2016 to May 2017 via an electronic survey. This two-region survey consisted of 19 questions (demographic data, knowledge and management of anaphylaxis, and management after acute episodes).

The questionnaire was returned by 18\% (44) of physicians, fifty percent of whom were female physicians. Most respondents had less than 5 years' experience, and about one third with more than eleven years; $77.3 \%$ of respondents worked in general hospitals, $70.5 \%$ in ED, 59.1\% in prehospital and $40.9 \%$ in an emergency call centre.
Among the respondents, $84.1 \%$ knew the recommendations from the French Society for Anaesthesia and Resuscitation (4). The European or American recommendations were less known (figure 1). A total of $47.7 \%$ (21) of physicians knew the classification of Ring and Messmer, while 15.9\% of respondents were not knowledgeable in any classification.

The recognition of symptoms of anaphylaxis are summarised in figure 1. This recognition was overall good for both respiratory and cutaneous symptoms, whereas the rate of recognition was lower for abdominal symptoms and especially for diarrhoea and vomiting. A standard protocol was available for only $33.3 \%$ (14) of physicians.

When considering biological markers, measurement of tryptase and histamine rates was performed immediately in respectively $69.2 \%$ and $73.3 \%$ of patients, within the first hour in respectively $15.4 \%$ and $13.3 \%$, and along similar proportions beyond the first hour. Tryptase measurement was performed more than once by $12.2 \%$ (5) of physicians.

Regarding first-line treatment, cutaneous-mucosal symptoms were treated by oral antihistamines by $95.3 \%$ of physicians, 
Figure 1. Percentage of clinical symptoms identified as anaphylaxis symptoms.

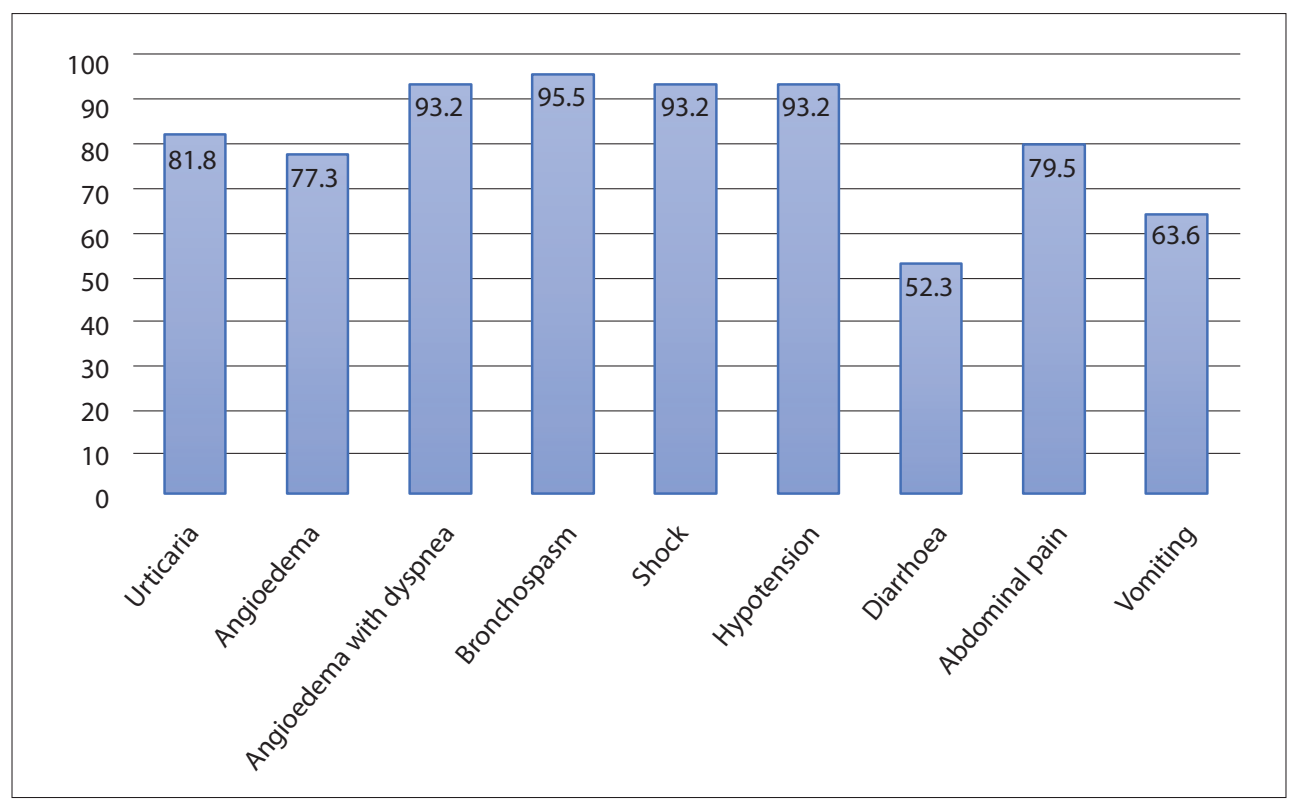

and by intravenous antihistamines or corticosteroids in $2.3 \%$. When symptoms were of grade 2 according to Ring and Messmer, intra-muscular epinephrine injection was administered by a mean $11.2 \%$ of physicians as first-line treatment, intravenous epinephrine injection by $8.4 \%$, antihistamines (oral route or IV) by $16.4 \%$ and corticosteroids (oral route or IV) by $17.8 \%$. For grade 3 symptoms, intramuscular epinephrine injection was performed by a mean $12.4 \%$ of physicians as first-line treatment, intravenous epinephrine injection by $64.3 \%$, intravenous antihistamines by $6.2 \%$ and corticosteroids (oral or intravenous route) by $16.6 \%$. First-line treatments for all grades are summarised in figure 2.

When considering management after an acute anaphylaxis episode, $2.3 \%$ of physicians provided for an allergy assessment, $86.4 \%$ never and $11.4 \%$ occasionally. For grade 2 reactions, $22.7 \%$ of physicians prescribed epinephrine self-injectors, and $52.2 \%$ for grade 3 . Over $88 \%$ of physicians reported not providing discharge documents to the patients while $86.4 \%$ did not refer patients to an allergy specialist.

The recognition of symptoms was good in the present study even for abdominal symptoms. However, several studies have shown that at least $50 \%$ of anaphylaxis episodes are misdiagnosed in the ED when current guidelines are not used $(5,6)$. This good recognition of symptoms contrasts with the fact that epinephrine was not given as first-line treatment and especially for grade 2 symptoms. Indeed, only $19.6 \%$ of physicians responded administering epinephrine as first-line treatment, regardless of route. This rate increased to $76.7 \%$ for grade 3 symptoms, although $6.2 \%$ of respondents answered using antihistamines as first-line treatment to counter these severe reactions. Another gap possibly reflecting the lack of standard protocols was that a standard protocol was only available for one-third of respondents. The lack of epinephrine use may also represent a low awareness of guideline recommendations or mistaken concerns regarding the efficacy and safety of intramuscular epinephrine $(7,8)$, of which several studies show similar results (9).

A recent retrospective study conducted by Corriger et al. in EDs in Lorraine, France, revealed interesting albeit somewhat discordant results with those of the present study (10). In their study, epinephrine use was reported in $7.5 \%$ of grade 2 and $32.4 \%$ of grade 3 reactions. The use of epinephrine by $76.7 \%$ of physicians in patients with grade 3 observed in our study was very high compared to the above study conducted in the same French region, although the discrepancy may be linked to differences in data collection methods. In Corriger et al., the data was collected by analysing the medical records selected by their ICM-10 code, with no specific code for anaphylaxis (10).

Measurement of tryptase rates within the first hour was performed by about two-thirds of our respondents. Despite recommendations, only $12.2 \%$ of respondents repeated this measurement more than once. This is likely related to the fact that these measurements are not useful for either acute diagnosis or management.

With regard to discharge recommendations, both provisions of written information and prescription of an epinephrine self-in- 
Figure 2. First-line treatment according to the Ring and Messmer severity scale.
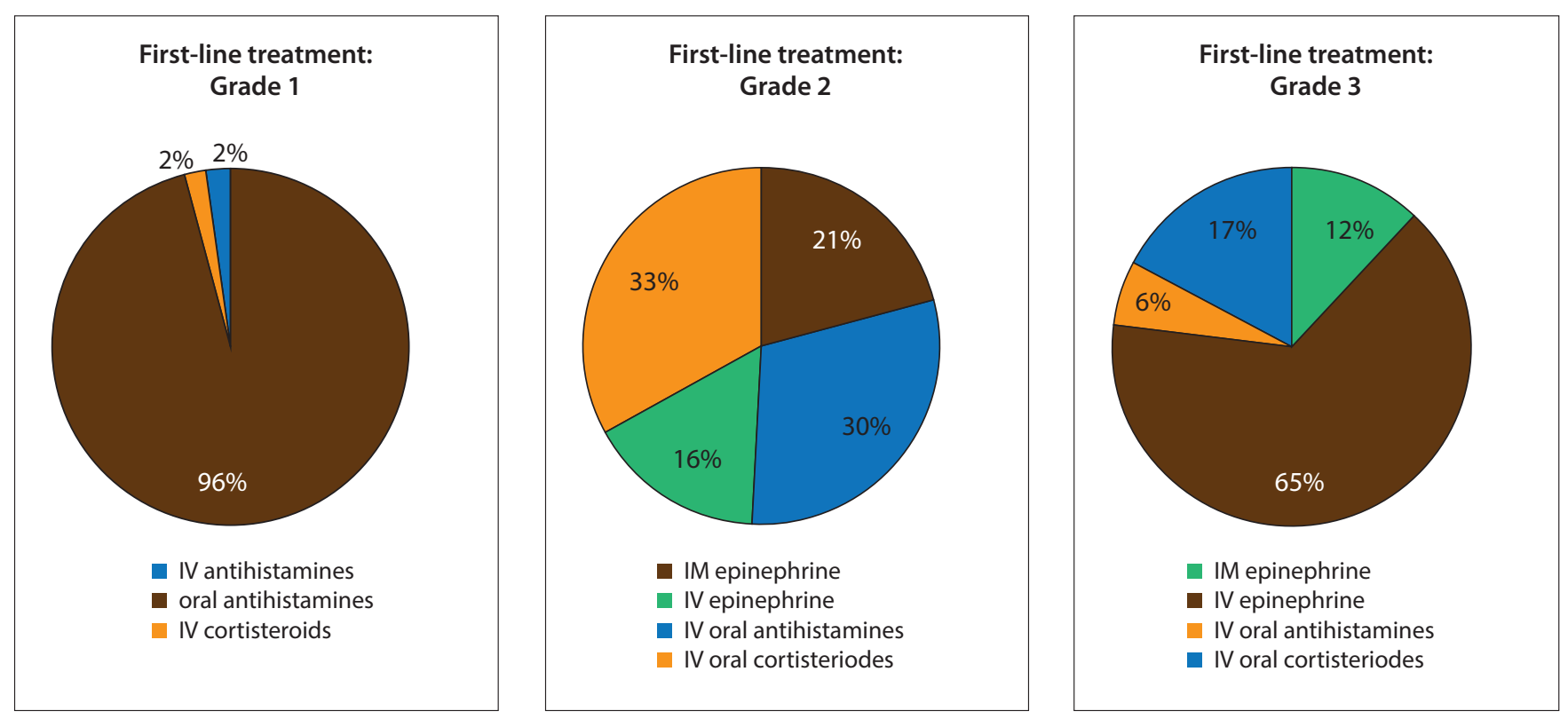

jector appeared inconsistent. Furthermore, the referral to an allergy specialist was rarely done. This may be indicative of certain difficulties in the collaboration with allergy centres, in order to complete diagnosis and treatment indications for prevention in subjects at risk.

In the study of Corriger et al. (10), tryptase measurement was performed in only $12.7 \%$ of patients, their data being similar to ours. Furthermore, the above study con-firmed the lack of epinephrine self-injector device prescriptions (17.3\%) and of referral for an allergy consultation (52.7\%) as demonstrated herein. In conclusion, to the best of our knowledge, this is the first survey to assess the concordance between the management of anaphylaxis in EDs and guideline recommendations in a French region. The outcomes confirm both the lack of epinephrine use in the $\mathrm{ED}$ as well as discharge indications.

As described in the literature, this poor use of epinephrine is likely the result of a lack of standard definition of anaphylaxis and of diagnostic difficulties.

The present data further underscore the need to strengthen collaboration between EDs and allergy centres. Lastly, this survey highlights the necessity for graduate and postgraduate courses on anaphylaxis in order to improve its management.

\section{References}

1. Muraro A, Roberts G, Worm M, Bilò MB, Brockow K, Fernández Rivas M, et al. Anaphylaxis: guidelines from the European Academy of Allergy and Clinical Immunology. Allergy 2014; 69(8):1026-1045.
2. Simons FER, Ardusso LR, Bilò MB, Cardona V, Ebisawa M, ElGamal YM, et al. Interna-tional consensus on (ICON) anaphylaxis. World Allergy Organ J 2014; 7(1):9.

3. Simons FER, Ebisawa M, Sanchez-Borges M, Thong BY, Worm M, Tanno LK, et al. 2015 update of the evidence base: World Allergy Organization anaphylaxis guidelines. World Allergy Organ J 2015; 8:32.

4. Mertes PM, Tajima K, Regnier-Kimmoun MA, Lambert M, Iohom G, Guéant-Rodriguez RM, et al. Perioperative anaphylaxis. Med Clin North Am 2010; 94(4):761-789, xi.

5. Harduar-Morano L, Simon MR, Watkins S, Blackmore C. Algorithm for the diagnosis of anaphylaxis and its validation using population-based data on emergency department visits for anaphylaxis in Florida. J Allergy Clin Immunol 2010; 126(1):98104.e4.

6. Gaeta TJ, Clark S, Pelletier AJ, Camargo CA. National study of US emergency department visits for acute allergic reactions, 1993 to 2004. Ann Allergy Asthma Immunol 2007; 98(4):360-365.

7. Arroabarren E, Lasa EM, Olaciregui I, Sarasqueta C, Muñoz JA, Pérez-Yarza EG. Improving anaphylaxis management in a pediatric emergency department. Pediatr Allergy Immunol 2011; 22(7):708-714.

8. Nowak R, Farrar JR, Brenner BE, Lewis L, Silverman RA, Emerman C, et al. Customizing anaphylaxis guidelines for emergency medicine. J Emerg Med 2013; 45(2):299-306.

9. Kastner M, Harada L, Waserman S. Gaps in anaphylaxis management at the level of physicians, patients, and the community: a systematic review of the literature. Allergy 2010; 65(4):435-444.

10. Corriger J, Beaudouin E, Rothmann C, Penven E, Haumonte Q, Thomas H, et al. Ana-phylaxis and emergency: Regional data in Lorraine and management [Internet]. 2017 Jan 11 [cited 2018 Feb 8]; Available from: http://www.em-consulte.com/en/article/1169293. 